\title{
A novel approach to treatment of neuromyelitis optica
}

A nonpathogenic monoclonal antibody might hold the key to successful treatment of neuromyelitis optica (NMO)

- an autoimmune demyelinating disease characterized by lesions in the optic nerve and spinal cord. In Annals of Neurology, Alan Verkman, Jeffrey Bennett and colleagues present a novel approach that targets the interaction between the NMO autoantibody (NMO-Ig) and its antigen, aquaporin-4 (AQP4).

"My group was responsible for the identification of the target of the NMO antibody in 1994," says Verkman. "NMO-Ig is very likely pathogenic by a mechanism involving astrocyte damage, which initiates a cascade of inflammation resulting in myelin loss and neurological deficit."

In the new study, AQP4-specific recombinant antibodies were cloned from the cerebrospinal fluid of patients with NMO. The researchers identified an antibody that bound with high affinity to AQP4, and they mutated the Fc portion of this antibody to eliminate its effector functions, thereby rendering it nonpathogenic.

The antibody was shown to block the binding of NMO-Ig to AQP4-expressing cells, and to protect against complementmediated cytotoxicity induced by serum from patients with NMO. Additional experiments performed in ex vivo
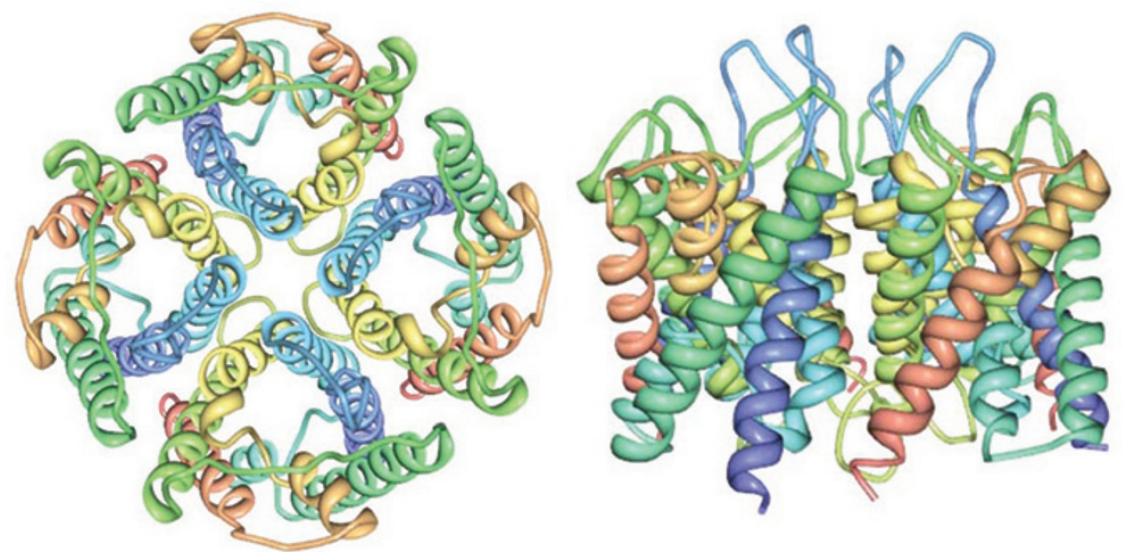

Aquaporin-4, the neuromyelitis optica autoantigen. Adapted from Jarius, S. \& Wildemann, B. AQP4 antibodies in neuromyelitis optica: diagnostic and pathogenic relevance. Nat. Rev. Neurol. 6, 383-392 (2010).

spinal cord slice cultures and an in vivo mouse model revealed that the antibody prevented the formation of NMO lesions in response to $\mathrm{NMO}-\mathrm{Ig}$.

"The idea that a nonpathogenic antibody can compete off a pathogenic antibody at a target is a novel concept in the therapy of autoimmune disease," explains Verkman.

"In NMO, it is particularly attractive because it affects the root cause of NMO rather than downstream cytotoxicity and inflammation."

The researchers are now planning further preclinical characterization and testing of their antibody, and they hope to develop it as a therapy for NMO. "We have filed a patent application and are discussing development with pharmaceutical companies," says Bennett. "We are continuing to optimize the blocking antibody, and are using other recombinant antibodies in high-throughput assays to identify small-molecule blockers of NMO-IgG binding to AQP4."

Heather Wood

Original article Tradtrantip, L. et al. Anti-aquaporin-4
monoclonal antibody blocker therapy for neuromyelitis
optica. Ann. Neurol. doi:10.1002/ana.22657
Further reading Zhang, H. et al. Ex vivo spinal cord
slice model of neuromyelitis optica reveals novel
immunopathogenic mechanisms. Ann. Neurol.
doi:10.1002/ana.22551

